\title{
Influence of Agaricus Bisporus Establishment and Fungicidal Treatments on Casing Soil Metataxonomy During Mushroom Cultivation
}

\section{Maria Luisa Tello ( $\sim$ biotecnologia@ctich.com )}

Mushroom Technological Research Center of La Rioja (CTICH)

\section{Rebeca Lavega}

Mushroom Technological Research Center of La Rioja (CTICH)

Margarita Pérez

Mushroom Technological Research Center of La Rioja (CTICH)

\section{Antonio J. Pérez}

Universidad Pablo Olavide (Sevilla)

\section{Michael Thon}

Universidad de Salamanca, Instituto Hispano-Luso de Investigaciones Agrarias (CIALE)

\section{Ernesto Pérez}

Universidad de Salamanca, Instituto Hispano-Luso de Investigaciones Agrarias (CIALE)

\section{Research Article}

Keywords: Mushroom, metataxonomy, microbiome, Agaricus, QIIME2, 16S, ITS.

Posted Date: November 15th, 2021

DOI: https://doi.org/10.21203/rs.3.rs-956209/v1

License: (c) (i) This work is licensed under a Creative Commons Attribution 4.0 International License. Read Full License 


\section{Abstract}

The cultivation of edible mushroom is an emerging sector with a potential yet to be discovered. Unlike plants, it is a less developed agriculture where many studies are lacking to optimize the cultivation. Mushrooms are a source of resources still to be revealed, which have applications not only in food, but in many other sectors such as health, industry and biotechnology. Mushroom cultivation consists of the development of selective substrates through composting where the mushroom grows via solid fermentation process. In case of Agaricus bisporus, the compost fully colonized by mycelium hardly produces mushrooms and it is necessary to apply a casing layer with certain physical, chemical and biological characteristics to shift from the vegetative mycelium to the reproductive one, where the native microbiota plays crucial roles. Currently, the industry faces a challenge to substitute the actual peat based casing materials due to the limited natural resources and the impact on the peatlands where peat is extracted.

In this work we have employed high-throughput techniques by next generation sequencing to screen the microbial structure of casing soil employed in mushroom cultivation while sequencing V3-V4 of the 16S rRNA gene for bacteria and the ITS2 region of rRNA for fungi in an Illumina MiSeq. In addition, the microbiome dynamics and evolution (bacterial and fungal communities) in peat based casing along the process of incubation of Agaricus bisporus have been studied, while comparing the effect of fungicidal treatment (Chlorothalonil and Metrafenone). Statistically significant changes in populations of bacteria and fungi were observed. Microbial composition differed significantly based on incubation day, changing radically from the original communities to a specific microbial composition adapted to enhance the $A$. bisporus mycelium growth. Chlorothalonil treatment seems to delay casing colonization by A. bisporus. Proteobacteria and Bacteroidota appeared as the most dominant bacterial phyla. We observed a great change in the structure of the bacteria populations between day 0 and the following days. Fungi populations changed more gradually, A. bisporus displacing the rest of the species as the cultivation cycle progresses. A better understanding of the microbial communities in the casing will hopefully allow us to increase the biological efficiency during production as well as possibly help us to have a clearer view of the microbial community-pathogen relationships as they are directly related to disease development.

\section{Background}

Mushrooms have been cultivated since ancient times especially in the eastern countries [1]. They have been appreciated not just for their flavour but for their nutritional and medicinal value. About 7,000 species of mushrooms are believed to have varying degrees of edibility, and more than 3,000 species of 231 different genera are considered to be major edible mushrooms [2]. Despite this, only about 60 edible mushrooms are grown commercially, and around 10 are produced on an industrial scale. Agaricus bisporus is cultivated all over the world for the production of button mushrooms. These carpophores have relative high protein content and contain fiber, vitamins, minerals and bioactive compounds. $A$. bisporus is grown in compost consisting of wheat straw, horse or chicken manure [3]. After the compost is colonized, it is covered with a casing soil, which is necessary for moisture and microbial flora. $[4,5,6]$. This casing soil is prepared with black or white peat and calcium carbonate, to reach $\mathrm{pH}$ 7-7.5. Peat is a fossil organic material, coming from deposits of vegetal organic matter (moss) accumulated over thousands of years. The use of peat in A. bisporus cultivation is essential, since until now no other material is known to have its characteristics: high water retention but water availability for the mushroom, porous and heavy structure and a specific microbiota [7]. Peat used for mushroom growing accounts for only a small portion of the total volumes of peat extracted worldwide. However, peat is an unsustainably sourced nonrenewable resource whose availability is diminishing due to reserve depletion and regulatory restrictions. Many attempts have been made to replace peat by other materials such as pine bark or coconut fibre. However, due to the particular physico-chemical properties and native microbiota, peat is still seen as the only optimal option [8]. Casing soil characteristics may also affect the development of mushroom diseases, such as dry bubble caused by Lecanicillium fungicola (Preuss), Zare and Gams, and wet bubble caused by Mycogone perniciosa (Magnus) Delacroix [9, 10]. To stop disease appearance, chemical products are applied. These are not specifically developed for mushroom pathogens since 
the sector is too small compared with other agricultural sectors. The consequences for the mushroom industry are problems such as non-specificity, resistance of pathogens and limitations in its use.

In order to improve mushroom cultivation methods and to find alternative peat materials compatible with the current environmental challenges, microbial communities in the casing soil must be determined and analysed. This knowledge could help in the development of artificial peat materials of non-fossil origin, more friendly to the environment and with inhibiting properties of other pathogenic organisms, thus improving both the cultivated mushroom sector and other agricultural sectors that use peat, as nurseries or gardens.

In this work we have employed high-throughput techniques by next generation sequencing (NGS) to screen the microbiome dynamics and evolution (bacterial and fungal communities) in peat based casing soil along the process of fructification of $A$. bisporus, and the impact of fungicidal treatment (Chlorothalonil and Metrafenone) on the microbiome communities. To reach these objectives two commonly used markers were sequenced in an Illumina MiSeq: V3-V4 of the 16S rRNA gene for bacteria and the ITS2 region of rRNA for fungi.

\section{Methods}

\section{Casing sampling}

Table 2. Samples utilized in the present work.

\begin{tabular}{|lll|}
\hline Cod. & date & Samples \\
\hline G1 & $14 / 01 / 2019$ & Casing soil mix (50\%blond peat, 50\% black peat) \\
\hline G2 & $18 / 01 / 2019$ & Casing at day 4* \\
\hline G3 & $21 / 01 / 2019$ & Casing at day 7 (Control) \\
\hline G4 & $21 / 01 / 2019$ & Casing at day 7 (Chlorothalonil) \\
\hline G5 & $21 / 01 / 2019$ & Casing at day 7 (Metrafenone) \\
\hline G7 & $30 / 01 / 2019$ & Casing at day 16 (Control) \\
\hline G8 & $30 / 01 / 2019$ & Casing at day 16 (Chlorothalonil) \\
\hline G9 & $30 / 01 / 2019$ & Casing at day 16 (Metrafenone) \\
\hline
\end{tabular}

*Samples at day 4 were taken before fungicide application.

Cultivation room was filled with phase III compost from a commercial mushroom compost yard (Germinados de Lodosa, L.S., Lodosa, Spain). The A. bisporus strain used was Sylvan A15. The casing soil used was $50 \%$ black peat and $50 \%$ blond peat from Valimex S.L. Three biological replicates of the casing samples were destructively taken from the cultivation room along the casing incubation process, from the peat-based casing at four different days (Table 2): casing day (D00), fungicides application day (D04), ruffling day (D07) and at beginning of first flush (D16). Fungicide treatments were applied in separated areas. Chlorothalonil treatment concentration was $3 \mathrm{ml} / \mathrm{m}^{2}$ and Metrafenone treatment application was $1 \mathrm{ml} / \mathrm{m}^{2}$.

\section{Total DNA extraction}


Three biological replicates of the casing samples were studied by extracting genomic DNA ( $\mathrm{n}=3$ replicates per sample type). Fresh samples were homogenized in a ceramic mortar with liquid nitrogen. DNA was extracted from up to $0.5 \mathrm{~g}$ of casing with NucleoSpin ${ }^{\circledR}$ Soil kit (MACHEREY-NAGEL). DNA quantity and quality were checked using $2 \mu$ of the purified template in a Qubit 2.0 Fluorometer and the Qubit dsDNA BR Assay kit (Thermo Fisher Scientific, MA, USA) and finally it was visualized on a 1.5\% agarose gel stained with Midori-Green Advance staining (Nippon Genetics, Tokyo, Japan).

\section{PCR amplification}

Library preparation for 16S rRNA and ITS2 gene amplicon sequencing was performed separately following the Illumina (San Diego, CA, USA) recommendations with some modifications. A 2-step amplification procedure was used [19] using paired end universal bacterial primers [20] for the V3-V4 hypervariable region of the 16S rRNA gene. For the hypervariable region ITS2, the primers ITS3 and ITS4 were used [21]. The primers used contained the Illumina sequencing adapters (overhang nucleotide sequences) added to the gene-specific sequences (Table 3).

Table 3. Primers used for bacteria and fungi sequences amplification.

\begin{tabular}{|lll|}
\hline V3- & Forward sequence $\left(5^{\prime} \rightarrow 3^{\prime}\right)$ & TCGTCGGCAGCGTCAGATGTGTATAAGAGACAGGCATCGATGAAGAACGCAGC \\
V4 & & \\
rRNA & Reverse sequence $\left(5^{\prime} \rightarrow 3^{\prime}\right)$ & GTCTCGTGGGCTCGGAGATGTGTATAAGAGACAGTCCTCCGCTTATTGATATGC \\
$\begin{array}{lll}\text { ITS2 } \\
\text { rRNA }\end{array}$ & Forward sequence $\left(5^{\prime} \rightarrow 3^{\prime}\right)$ & TCGTCGGCAGCGTCAGATGTGTATAAGAGACAGCCTACGGGNGGCWGCAG \\
\cline { 2 - 3 } & Reverse sequence $\left(5^{\prime} \rightarrow 3^{\prime}\right)$ & GTCTCGTGGGCTCGGAGATGTGTATAAGAGACAGGACTACHVGGGTATCTAATCC \\
\hline
\end{tabular}

PCR amplification were performed with the KAPA HiFi HotStart ReadyMix PCR Kit in a T100 Thermal Cycler (BioRad) under the following conditions: $95^{\circ} \mathrm{C}$ for $120 \mathrm{~s}$, followed by 25 cycles of $95^{\circ} \mathrm{C}$ for $30 \mathrm{~s}, 55^{\circ} \mathrm{C}$ for $30 \mathrm{~s}, 72{ }^{\circ} \mathrm{C}$ for $30 \mathrm{~s}$ and a final cycle of $72{ }^{\circ} \mathrm{C}$ for $5 \mathrm{~min}$. The presence of the amplified $16 \mathrm{~S}$ rRNA and the ITS gene bands was verified in $1.5 \%$ agarose gels. PCR products were purified using NucleoMag NGS Clean-up and Size Select (Macherey- Nagel Co., Diiren, Germany) and eluted in $50 \mu \mathrm{L}$ of $10 \mathrm{mM}$ Tris pH8.5. After purification with the magnetic beads, $5 \mu \mathrm{L}$ of DNA was amplified in a second PCR employing Nextera XT Index primers (Illumina). This PCR was run at $95^{\circ} \mathrm{C}$ for 3 min, followed by 8 cycles of $95^{\circ} \mathrm{C}$ for $30 \mathrm{~s}, 55^{\circ} \mathrm{C}$ for $30 \mathrm{~s}, 72{ }^{\circ} \mathrm{C}$ for $30 \mathrm{~s}$ and a final cycle of $72{ }^{\circ} \mathrm{C}$ for $5 \mathrm{~min}$. A second purification step with NucleoMag magnetic beads was carried out after the Nextera indexing PCR. The 16S V3-V4 rRNA and the ITS2 gene amplicons containing the Nextera indexes were finally eluted in $25 \mu \mathrm{L}$ of $10 \mathrm{mM}$ Tris pH 8.5. Resulting libraries were quantified by Qubit® 2.0 (Thermo Fisher Scientific) and qualified by Fragment Analyzer (Advanced Analytical Technologies) [14].

\section{Library pooling and sequencing}

Both libraries (one for bacteria and one for fungi samples) were created by pooling $5 \mu \mathrm{L}$ at $4 \mathrm{nM}$ concentration of each sample. Each pool was sequenced separately. Pools were then diluted with hybridization buffer to 12 pM, using $15 \%$ PhiX as a control for low-diversity libraries. Sequencing was performed on an Illumina MiSeq sequencer based in CTICH, using a 2 x 300 pb paired-end run (MiSeq Reagent kit v3 (MS-102-3001) following the manufacturer's instructions (Illumina, Inc., San Diego, CA, USA).

\section{Sequence analyses}

Sequencing data were demultiplexed using Illumina bcl2fastq® program. Demultiplexed paired FASTQ sequences were imported in QIIME2 artifact format and analysed with QIIME2 v2020.8. Used workflow is described in detail in the following link: https://github.com/Marylou8/Metataxonic-analysis-using-Qiime2-workflow. Quality control was carried out using the DADA2 pipeline [22] incorporated into QIIME2 [23,24]. The DADA2 program filtered out PhiX reads, removed 
chimeric sequences and assigned reads into Amplicon Sequence Variants (ASVs). Taxonomic annotation for bacteria was obtained using SILVA v138 database [25]. Taxonomic annotation for fungi was obtained using UNITE v8.2 2020 database [26]. Chloroplast and mitochondrial contaminants were detected and filtered using the QIIME2 "taxa filter-table" and "taxa filter-seqs" commands.

To filter out low-abundance features, we follow the approach of Morton (https://forum.qiime2.org/t/ancom-givingstrange-w-values/1002/11) where those features which do not sum at least 10 sequences among all samples, as well as those that only appear in one sample were filter out (command "feature-table filter-features"). Differential abundance analysis was analysed with ANCOM test [27]. The data-sets will be submitted to National Center for Biotechnology Information (NCBI).

\section{Statistics}

Sequencing statistical analyses were done using QIIME2 v2020.8 [28]. Alpha diversity (within-samples) was analysed using Faith's phylogenetic diversity: bacterial community richness that incorporates phylogenetic relationships between taxa [29], Pielou's species evenness: bacterial community evenness [30] and Shannon's index: bacterial community richness and evenness [31]. To determine significance in alpha diversity, non-parametric Kruskal-Wallis comparisons were performed [32]. Box-and-whisker plots for species richness and evenness were generated using QIIME2. Alpha rarefaction curves were analysed, to assess if sampling depth was enough to observe the full community diversity [33]. Beta diversity was analysed using Bray-Curtis distance (abundance without phylogeny) [34] and unweighted UniFrac distance (presence and absence of OTUs with phylogeny) [35]. Principle Coordinate Analysis (PCoA) plots were generated from Bray-Curtis distances and unweighted UniFrac distance using QIIME2. To generate taxonomy heatmaps R-Studio Version 1.3.1093 was used [36]. Two libraries were necessary to plot the heatmaps from QIIME2 artifacts "tidyverse" [37] and "qiime2R" [38].

\section{Results}

Data from the trial were divided in three data-sets. The first one (data-set DAYS) represents the differences between incubation days in the control without fungicides application: casing day (D00), fungicides application (D04), ruffling day (D07) and beginning of first flush (D16). Ruffling is an agronomic technique used in mushroom farming that consists of raking the surface of the casing soil in order to aerate it and mix compost with the casing so that mushroom colonization is faster and more homogeneous. The second (data-set TREATMENTS D07) represents the differences between the control and the two fungicide treatments (Chlorothalonil and Metrafenone) at ruffling day (D07). And finally, the third (data-set TREATMENTS D16) represents the differences between the control and the two fungicide treatments in the beginning of the first flush (D16). For these three data-set both bacterial and fungi communities have been analysed.

\section{Sequencing and assembly}

\section{Bacterial $16 S$ analysis}

For both, the Bacterial 16S analysis and the Fungi ITS2 analysis, all samples $(n=24)$ were sequenced on the Illumina MiSeq. Sample 26 (G9) from the Bacterial 16S analysis, was excluded from the analysis because of the low number of reads obtained (1168 reads). All data from the metataxonomic analysis are collected in Table 1.

Table 1. Metataxonomic analysis data. 


\begin{tabular}{lll} 
Analysis & Bacterial 16S & Fungi ITS2 \\
\hline Number of samples & 23 & 24 \\
\hline Total read pairs & 3935266 & 8407044 \\
\hline Total read pairs average & 178875 & 350293 \\
\hline Total read pairs SD (+/-) & 68328 & 262317 \\
\hline Truncation selected (forward/reverse) & $260 / 230$ & $280 / 200$ \\
\hline ASV's classified after DADA2 & 14018 & 2533 \\
\hline ASV's classified after filtering & 3074 & 950
\end{tabular}

Alpha rarefaction curves were analysed (supplementary material Figure 7 and 8), to confirm that sampling depth was enough to observe the full community diversity. Alpha and beta diversity box-and-whisker plots and statistics are collected in Supplementary material Figures 7, 8, 9, 10, 11 and 12).

\section{Analysis data-set DAYS}

First we performed a taxomonic analysis to study the different bacterial populations. Phylum and family level were visualized in barplots. Then, we analysed the diversity to check if there were significant differences within samples (alpha diversity) and between samples (beta diversity). Finally, we carried out an ANCOM test to identify different abundances across bacterial samples at genus level.

The most highly represented bacteria phylum were Proteobacteria and Bacteroidota for all samples in data-set DAYS (Fig. 1A). Proteobacteria at D00 appears at 40.34\%+/-1.39 SD (percentage of ASVs classified) and increases along days, with a percentage of $53.70 \%+/-0.96$ SD at D16. Firminutes phylum presents a $10.88 \%+/-0.88$ SD the first day (D00), decreasing along the cycle until it reaches a percentage of $1.24 \%+/-0.01$ SD D16. Other phyla present in smaller percentages, such as Patescibacteria, Verrucomicrobiota, Chloroflexi and Planctomycetota, increased during the days. One candidate phylum (WPS-2=Eremiobacterota) and one phylum without identification was found.

At family level differences between days can be observed (Fig. 1B). Day D00 appears to have more differences comparing to the other three days. Most abundant family at day D00 was Bacteroidaceae, with a $6.70 \%+/-1.03$ SD. This family decreased deeply during the cycle until D16 with a $0.13 \%+/-0.11$ SD. In addition, family Lachnospiraceae almost disappeared at the end of the cycle. Family Xanthomonadaceae was present in all samples with a similar percentage (4$6 \%$ ). However, the presence of some families increased during days, such as Sphingomonadaceae (from 1.36\% $+/-0.47$ SD to $7.99 \%+/-0.70$ SD), Chitinophagaceae (from 1.45\% +/- 0.34 SD to 5.03\% +/-0.14 SD), Devosiaceae (from 0.94\% $+/-0.07$ SD to $2.86 \%+/-0.74$ SD) or Spirosomaceae $(0.20 \%+/-0.06$ SD to $2.25 \%+/-0.20$ SD).

Alpha diversity results showed that there were not significant differences between all days according to Faith's phylogenetic diversity $(p=0.059)$ and Pielou's species evenness $(p=0.053)$. Bacterial community richness and evenness showed significant differences by Shannon's index $(p=0.027)$. However, results showed higher diversity with-in samples at day 4 (D04) and lower at day zero (D00). Beta diversity results showed significant differences by Bray Curtis: abundance without phylogeny $(p=0.001)$ and unweighted UniFrac distance: presence and absence of OTUs with phylogeny $(p=0.001)$.

An ANCOM test was also carried out, to compare the composition of microbiomes in the bacterial populations. Results identified 19 different abundances across samples by days at genus level. Aquicella, Halomonas, Solitalea, Lacihabitans, Caulobacter and Anaerosporobacter had the highest W value (Supplementary material Table S1). 
The most identified fungal phyla belong to Ascomycota and Basidiomycota. Unfortunately, an important number of sequences were not classified in any phylum (Fig. 2A). At day zero (D00) the most abundant phylum was Ascomycota (69.13\%+/-0.27 SD). This tendency changes along the cultivation cycle with phylum Ascomycota decreasing D04 (57.90\%+/-2.87 SD), D07 (18.68\%+/-7.22 SD) until D16 with 3.86\%+/-1.7 SD. Phylum Basidiomycota increases from D00 $(22.38 \%+/-0.10 \mathrm{SD})$ until D16 (91.95\% +/-3.29 SD), becoming the controlling phylum from D07. This trend can be also observed at Family level (Fig. 2B), the Agaricaceae increasing from D00 (2.44\%+/-0.73 SD) to the D16 (89.43\%+/-4.33 $\mathrm{SD})$.

At family level we can observe clearly the change in the populations of the different fungi families. Family Agaricaceae displaced the other families across the cycle while A. bisporus is growing. Helotiaceae family in the beginning was present in an important percentage $(18.74 \%+/-4.29$ SD) that almost disappears at D16 $(0.54 \%+/-0.13$ SD), as well as other families such as Piskurozumaceae, Exidiaceae or Myxotrichaceae. On the other hand, the presence of the family Aspergillaceae increased from D00 (13.54\%+/-6.32 SD) to the D04 (28.84\%+/-1.23 SD) and then it decreased D07 and D16 (1.58\%+/-0.94 SD).

Alpha diversity results showed that there were significant differences between all days using Faith's diversity $(p=0.024)$, Pielou's evenness $(p=0.023)$ and Shannon's index $(p=0.024)$. Beta diversity results showed also significant differences by Bray Curtis: abundance without phylogeny $(p=0.001)$ and unweighted UniFrac distance $(p=0.001)$.

ANCOM test results identified different abundance across samples by days at genus level in genus Agaricus (Supplementary material Table S2).

\section{Analysis data-set TREATMENTS D07}

Two different fungicidal treatments were applied in the trial: Chlorothalonil and Metrafenone. Also, samples from a Control without any treatment were analysed. The most representative bacterial phyla were Proteobacteria and Bacteroidota for all samples in data-set TREATMENTS D07 (Fig 3A). The highest Proteobacteria abundance corresponds to Chlorothalonil treatment with $48.51 \%+/-1.84$ SD. Three candidate phyla were found, WPS-2, WS2 and FCPU426. Most abundant families are common in the three treatments (Fig 3B), such as Flavobacteriaceae (89\%), Xanthomonadaceae (6.7-6.9\%) and Sphingomonadaceae (6.8-5.3\%). Family Chitinophagaceae showed higher abundance in Metrafenone treatment (5.4\% +/-.036 SD) and lower in Chlorothalonil treatment (4.51\% +/-0.32 SD). Other families such as Rhizobiaceae had a higher abundance in the control ( $2.64 \%+/-0.43 \mathrm{SD})$ than with the treatments (Chlorothalonil $1.90+/-0.70$ SD and Metrafenone 1.02\% +/-0.54 SD). Family Rhodanobacteraceae presence is $1 \%$ higher with Metrafenone treatment $2.42 \%+/-0.13$ SD.

Alpha diversity results showed that there were not significant differences between all treatments using Faith's diversity $(p=0.956)$, Pielou's evenness $(p=0.875)$ and Shannon's index $(p=0.956)$. Beta diversity results showed no significant differences by Bray Curtis: abundance without phylogeny $(p=0.149)$ and unweighted UniFrac distance $(p=0.237)$.

ANCOM test results did not identified different abundances across samples by treatments.

For the fungal phylum level (Fig. 4A) we observe that in the Control without treatment, Basidiomycota was the most abundant phylum with $69.71 \%+/-10.22$ SD. For Chlorothalonil treatment both phyla were in similar proportions: Basidiomycota 38.55\%+/-4.95 SD and Ascomycota 38.33\%+/-11.88 SD. For this treatment phylum Mucoromycota was more abundant than in the other 2 treatments. For Metrafenone treatment the most abundant phylum was Basidiomycota with $68.43 \%+/-7.20$ SD.

At family level there were around $10-20 \%$ of sequences unidentified (Fig. 4B). Family Agaricaceae in Control and with Metrafenone treatment had a high presence $(63.48 \%+/-11.15$ SD and $61.88 \%+/-9.41$ SD) while in Chlorothalonil 
treatment the percentage of this family was just $26.09 \%+/-8.04$ SD. The other two families identified with an important presence were Aspergillaceae and Piskurozymaceae.

Alpha diversity results showed that there were not significant differences between all treatments using Faith's diversity $(p=0.066)$, Pielou's evenness $(p=0.060)$ and Shannon's index $(p=0.060)$. Beta diversity results showed significant differences by Bray Curtis: abundance without phylogeny $(p=0.013)$ and unweighted UniFrac distance $(p=0.005)$.

ANCOM test results do not identified different abundance across samples by treatment at genus level.

\section{Analysis data-set TREATMENTS D16}

Similar phylum abundance was found for the data-set TREAMENTS D16 (Fig. 5A). Proteobacteria phylum showed also the highest presence in all samples, representing more than $50 \%$ of the bacteria identified. Also for this data-set, Proteobacteria was more abundant in Chlorothalonil treatment with 54.78\%+/-2.34 SD. At family level (Fig. 5B) the most common families in all samples were Sphingomonadaceae $(8.69 \%+/-0.84$ SD at Control, 9.03\%+/-2.26 SD with Chlorothalonil and 4.54\%+/-1.29 SD with Metrafenone treatment), Chitinophagaceae, Comamonadaceae, Flavobacteriaceae and Rhizobiaceae.

Alpha diversity results showed that there were not significant differences between all treatments using Faith's diversity $(p=0.235)$, Pielou's evenness $(p=0.367)$ and Shannon's index $(p=0.986)$. Beta diversity results showed significant differences by Bray Curtis: abundance without phylogeny $(p=0.019)$ and unweighted UniFrac distance $(p=0.011)$.

ANCOM test results identified different abundances across samples by treatments at genus level. There were 5 genera identified: "uncultured", Pirellula, Vicingus, 37-13 and Sandaracinus (Supplementary material Table S3).

In this data-set fungal phylum Basidiomycota was the most abundant in all treatments (Fig. 6A). However, differences in the abundance could be observed. In case of Metrafenone treatment, Basidiomycota appeared in a 70.72\%+/-22.34 SD and Ascomycota at 15.35\%+/-15.29 SD. These standard deviations were higher than in the other treatments. For Chlorothalonil treatment, Basidiomycota phylum had a presence of 76.29\%+/-7.91 SD and for the Control Basidiomycota had the highest occurrence with 91.95\%+/-3.29 SD.

At family level Agaricaceae was the dominant family (Fig. 6B). However, its presence was lower in the treatments (Chlorothalonil 69.37\%+/-13.65SD and Metrafenone 63.28\%+/-28.38 SD) comparing to the Control (89.43\%+/-4.33 SD). In this data-set there were also a high number of unidentified sequences. Other families with an important presence like in the data-set TREATMENT D07 were Aspergillaceae and Piskurozymaceae.

Alpha diversity results showed that there were not significant differences between all treatments using Faith's diversity $(p=0.670)$, Pielou's evenness $(p=0.193)$ and Shannon's index $(p=0.288)$. Beta diversity results showed not significant differences by Bray Curtis: abundance without phylogeny $(p=0.324)$ and unweighted UniFrac distance $(p=0.641)$.

ANCOM test results do not identified differences in abundance across samples by treatment at genus level.

\section{Discussion}

The goal of our research is to understand the changes in the microbial dynamics that occur during mycelium development and mushroom (A. bisporus) establishment in the casing soil, and the influence of fungicide treatment application on the microbial population changes of this active ecosystem.

The structure of the populations of both bacteria and fungi, at the beginning of the culture (day zero= D00), fluctuate with respect to the other days. These data confirm that the casing soil in contact with the compost colonized by A. bisporus, 
undergoes changes in the dynamics of its populations. In agreement with other research on A. bisporus, we observed that the predominant bacterial phylum identified in all the casing soil samples was Proteobacteria, followed by Bacteroidota $[11,12]$. Proteobacteria population increased along the days as seen in other studies [13], and Firmicutes phylum decreased throughout the cycle. Other phyla present in smaller percentages, such as Patescibacteria, Verrucomicrobiota, Chloroflexi and Planctomycetota, increased during the days. Most abundant family at day D00 was Bacteroidaceae, which decreased deeply during the cycle, like family Lachnospiraceae that almost disappeared at the end of the cycle. However, the presence of some families increased during days, such as Sphingomonadaceae, Chitinophagaceae, Devosiaceae or Spirosomaceae. Results identified different abundances at genus level across samples by days in 19 genera. The genera with highest W value were Aquicella, Halomonas, Solitalea, Lacihabitans, Caulobacter and Anaerosporobacter. These genera were not present D00 and they started to grow to significant populations during the incubation. Between day D07 and D16 a change in family's abundance was observed. Flavocteriaceae and Xanthomonadaceae had a higher presence D07 while Sphingomonadaceae and Chitinophagaceae at D16. At genus level, Flavobacterium was the most abundant in all samples and Pseudomonas increase along days as has been suggested in other research [14]. There were 5 genera identified with different abundances between treatments at D16: "uncultured", Pirellula, Vicingus, 37-13 and Sandaracinus. The most abundant bacterial species identified (Supplementary material Fig. 15) at day D00 was Bacteroides graminisolvens, a xylanolytic bacterial strain [15] that could be related with the decomposition of wheat straw in the casing soil development process. This genus almost disappears along the incubation days.

At day D00 the most abundant fungal phylum was Ascomycota, but throughout the cultivation cycle, the phylum Basidiomycota became dominant as described in other studies $[16,17]$. As indicated in these works, $A$. bisporus replace the other species and colonized the casing soil material. For this reason, from day D16 it becomes very difficult to determine the presence or changes in the populations of other fungi. Families Helotiaceae, Piskurozumaceae, Exidiaceae or Myxotrichaceae were present in an important percentage at D00 and almost disappears at D16. On the other hand, Aspergillaceae family presence increased from D00 to D4 and then it decreased at days D07 and D16. At D07 for the Control without treatment, Basidiomycota was the most abundant phylum while for Chlorothalonil treatment both Basidiomycota and Ascomycota were in similar proportions. For Chlorothalonil treatment phylum Mucoromycota was more abundant than in the other 2 treatments. For Metrafenone treatment the most abundant phylum was Basidiomycota. Family Agaricaceae in Control and with Metrafenone treatment had a high presence while in Chlorothalonil treatment the presence of this family is lower. The other two families identified with an important presence were Aspergillaceae and Piskurozymaceae. At D07 we observed an effect of Chlorothalonil treatment on the development of $A$. bisporus, being lower its presence in the treated samples (Supplementary material Fig. 19). Beta diversity results confirmed fungicide treatments effect on the bacterial and fungi populations. At D16 phylum Basidiomycota was the most abundant in all treatments. Metrafenone treatment had higher standard deviations than other treatments. At family level Agaricaceae was the dominant family being the Control treatment the one with the highest presence.

Fungal diseases were found in the samples. Lecanicillium genus was detected in samples at D00 and D04, but also in one sample at D07, treated with Chlorothalonil. A small number of sequences belonging to Mycogone perniciosa were found just in one sample at D00.

For the metataxonomy analysis with fungal ITS2, many sequences were assigned to "unidentified" taxon. These results were also found in other similar studies $[17,18]$ although they were focused on the compost dynamics and not the casing soil changes. Also it was not possible to reach species level for both bacteria and fungi, just for some sequences and species. There is still much work to do in taxonomic identification to build better and more complete databases.

\section{Conclusions}


The aim of the study was to better understand the changes experienced by different populations of fungi and bacteria in the casing soil used in mushroom production in order to improve mushroom cultivation and find more sustainable alternatives to peat based casing. This study shows that the use of QIIME2 is valid for NGS sequence analysis in this type of samples. However, the current databases do not allow us to reach the species level in most cases, just genus level. Significant differences have been found between the populations of both fungi and bacteria between the days of incubation, being more pronounced between the casing day (D00) and day 4 (D04). Differences have also been found between the fungicide treatments applied. Our improved understanding of the microbiology of casing soil provides renewed potential to design consortia of bacteria and fungi that could be used to optimize alternative casing materials. More detailed studies are also required to explore the relationship between microbial activity and diversity in casing soil and its implications in the mycelium and primordia development. Finally, a more thorough understanding of the pathogens control would have the potential to increase the quality and quantity of mushrooms produced.

\section{Abbreviations}

Amplicon Sequence Variant (ASV)

Analysis of Composition of Microbiomes (ANCOM)

Divisive Amplicon Denoising Algorithm (DADA)

National Center for Biotechnology Information (NCBI)

Next Generation Sequencing (NGS)

Polymerase chain reaction (PCR)

Principal Coordinates Analysis (PCoA)

Quantitative Insights Into Microbial Ecology version 2 (QIIME2)

Standard Deviation (SD)

\section{Declarations}

\section{Ethics approval and consent to participate}

Not applicable

\section{Consent for publication}

Not applicable

\section{Availability of data and materials}

The datasets analysed during the current study are available in the NCBI SRA repository, with the BioProject accession number PRJNA772891, https://www.ncbi.nlm.nih.gov/sra/PRJNA772891

\section{Competing interests}

The authors declare that they have no competing interests

\section{Funding}


This work was co-funded by the FEDER Operational Program La Rioja 2014-2020 (Project CT21_04) and the Department of Economic Development and Innovation of La Rioja. Res. nº 1168/2018.

\section{Authors' contributions}

MLT and RL performed the experiments and design the study. MLT, AJP, MT performed primary data analysis. MLT carried out bioinformatics analysis and wrote the manuscript. MP and EP participated in the design of the study and helped to draft the manuscript.

All authors read and approved the final manuscript.

\section{Acknowledgements}

The authors thank Dr. Jaime Carrasco (Marie Sklodowska-Curie grant agreement no. 742966) for the samples provided.

\section{Authors' information (optional)}

\section{Affiliations}

\section{Mushroom Technological Research Center of La Rioja (CTICH), Ctra. Calahorra km 4, 26560 Autol (La Rioja), Spain.}

Maria Luisa Tello, Rebeca Lavega and Margarita Pérez

Universidad de Salamanca. Instituto Hispano-Luso de Investigaciones Agrarias (CIALE). Calle Río Duero 12, 37185 Villamayor (Salamanca), Spain.

Michael Thon and Ernesto Pérez

Universidad Pablo Olavide (Sevilla), Spain.

Antonio J. Pérez

Corresponding author

Correspondence to Maria Luisa Tello.

\section{References}

[1] Chang S.T. and Hayes W.A. The biology and cultivation of edible mushrooms. Academic Press, New York, NY (1976).

[2] Chang S.T. and Wasser S.P., The role of culinary-medicinal mushrooms on human welfare with a pyramid model for human health, International Journal of Medicinal Mushrooms, vol. 14, no. 2, pp. 95-134, (2012).

[3] Pelkmans, J.F., Vos, A.M., Scholtmeijer, K. et al. The transcriptional regulator $\mathrm{c} 2 \mathrm{~h} 2$ accelerates mushroom formation in Agaricus bisporus. Appl Microbiol Biotechnol 100, 7151-7159 (2016). https://doi.org/10.1007/s00253-016-7574-9

[4] Bels-Koning H (1950) Experiments with casing soils, water supply and climate. Mushroom Sci 1:78-84

[5] Flegg P. The casing layer in the cultivation of the mushroom (Psalliota hortensis). J Soil Sci 7:168-176 (1956)

[6] Kalberer PP (1987) Water potentials of casing and substrate and osmotic potentials of fruit bodies of Agaricus bisporus. Sc Horticulturae 32:175-182 
[7] Pardo-Giménez, A., Pardo, J.E., and Zied, D.C. (2017) Casing materials and techniques in Agaricus bisporus cultivation. In Edible and Medicinal Mushrooms: Technology and Applications. D.C. Zied, and A. Pardo-Giménez (eds). Hoboken, NJ, USA: John Wiley \& Sons Ltd, pp. 385- 413.

[8] Carrasco J., Tello M.L., Perez M., Preston G. (2018) Biotechnological Requirements for the Commercial Cultivation of Macrofungi: Substrate and Casing Layer. In: Singh B., Lallawmsanga, Passari A. (eds) Biology of Macrofungi. Fungal Biology. Springer, Cham. https://doi.org/10.1007/978-3-030-02622-6_7

[9] Berendsen RL, Kalkhove SI, Lugones LG, Wösten HA, Bakker PA. Germination of Lecanicillium fungicola in the mycosphere of Agaricus bisporus. Environ Microbiol Rep 2012;4:227-233.

[10] Largeteau ML, Savoie JM. Microbially induced diseases of Agaricus bisporus: biochemical mechanisms and impact on commercial mushroom production. Appl Microbiol Biotechnol 2010;86:63-73.

[11] Siyoum, N. A., Surridge, K., van der Linde, E. J., and Korsten, L. 2016. Microbial succession in white button mushroom production systems from compost and casing to a marketable packed product. Ann. Microbiol. 66:151-164. https://doi.org/10.1007/s13213-015-1091-4 Crossref, ISI, Google Scholar

[12] Yang, W., Wang, L., Hu, Q., Pei, F., and Mugambi, M. A. 2019. Identification of bacterial composition in freeze-dried Agaricus bisporus during storage and the resultant odor deterioration. Front. Microbiol. 10:1-12. https://doi.org/10.3389/fmicb.2019.00349 ISI, Google Scholar

[13] Pecchia J, Cortese R, Albert I (2014) Investigation into the microbial community changes that occur in the casing layer during cropping of the white button mushroom, Agaricus bisporus. In: Singh M (ed) Proceedings of the 8th International Conference on Mushroom Biology and Mushroom Products, New Delhi, pp 309-313

[14] Carrasco, J., Tello, M. L., De Toro, M., Tkacz, A., Poole, P., Pérez-Clavijo, M., Preston, G. (2019). Casing microbiome dynamics during button mushroom cultivation: implications for dry and wet bubble diseases. Microbiology. DOI: 10.1099/mic.0.000792

[15] Nishiyama T., Ueki A., Kaku N., Watanabe K., Ueki K. (2009). Bacteroides graminisolvens sp. nov., a xylanolytic anaerobe isolated from a methanogenic reactor treating cattle waste. Int J Syst Evol Microbiol 59, 1901-1907.

[16] Carrasco, J., García-Delgado, C., Lavega, R., Tello, M.L., De Toro, M., Barba-Vicente, V., et al. (2020) Holistic assessment of the microbiome dynamics in the substrates used for commercial champignon (Agaricus bisporus) cultivation. J Microbial Biotechnol 13: 1933-1947

[17] McGee, C.F., Byrne, H., Irvine, A., and Wilson, J. (2017b) Diversity and dynamics of the DNA- and cDNA-derived compost fungal communities throughout the commercial cultivation process for Agaricus bisporus. Mycologia 109: 475-484.

[18] Zhang, X., Zhong, Y., Yang, S., Zhang, W., Xu, M., Ma, A., Zhuang, G., Chen, G. and Liu, W. (2014). Diversity and dynamics of the microbial community on decomposing wheat straw during mushroom compost production. Bioresource Technology, 170:183-195.

[19] Rocchi S, Valot B, Reboux G, Millon L. (2017) DNA metabarcoding to assess indoor fungal communities: Electrostatic dust collectors and Illumina sequencing. J Microbiol Methods;139:107-112.

[20] Klindworth A, Pruesse E, Schweer T, Peplies J, Quast C et al. Evaluation of general 16S ribosomal RNA gene PCR primers for classical and next-generation sequencing-based diversity studies. Nucleic Acids Res 2013;41:e1.

Page $12 / 18$ 
[21] White T, Bruns T, Lee S. Taylor J amplification and direct sequencing of fungal ribosomal RNA genes for phylogenetics. In: Innis M, Gelfand D, Shinsky J and White T (editors). PCR Protocols: a Guide to Methods and Applications. San Diego: Academic Press; 1990. pp. 315-322.

[22] Callahan, Benjamin J, Paul J McMurdie, Michael J Rosen, Andrew W Han, Amy Jo A Johnson, and Susan P Holmes, 'DADA2: High-Resolution Sample Inference from Illumina Amplicon Data', Nature Methods, 13 (2016), 581 <https://doi.org/10.1038/nmeth.3869>

[23] Caporaso, J. G. et al. QIIME allows analysis of high-throughput community sequencing data. Nat. Methods 7, 335336 (2010).

[24] Bolyen, E., Rideout, J.R., Dillon, M.R. et al. Reproducible, interactive, scalable and extensible microbiome data science using QIIME 2. Nat Biotechnol 37, 852-857 (2019). https://doi.org/10.1038/s41587-019-0209-9

[25] Quast C, Pruesse E, Yilmaz P, Gerken J, Schweer T, Yarza P, Peplies J, Glöckner FO (2013) The SILVA ribosomal RNA gene database project: improved data processing and web-based tools. Opens external link in new windowNucl. Acids Res. 41 (D1): D590-D596.

[26] Abarenkov, Kessy; Zirk, Allan; Piirmann, Timo; Pöhönen, Raivo; Ivanov, Filipp; Nilsson, R. Henrik; Kõljalg, Urmas (2020): UNITE QIIME release for Fungi 2. UNITE Community. 10.15156/BIO/786387

[27] Mandal S. et al.,(2015) Analysis of composition of microbiomes: a novel method for studying microbial composition. Microb Ecol Health Dis, 26:p. 27663.

[28] Bolyen E, Rideout JR, Dillon MR, Bokulich NA, Abnet CC, Al-Ghalith GA, Alexander H, Alm EJ, Arumugam M, Asnicar F, Bai Y, Bisanz JE, Bittinger K, Brejnrod A, Brislawn CJ, Brown CT, Callahan BJ, Caraballo-Rodríguez AM, Chase J, Cope EK, Da Silva R, Diener C, Dorrestein PC, Douglas GM, Durall DM, Duvallet C, Edwardson CF, Ernst M, Estaki M, Fouquier J, Gauglitz JM, Gibbons SM, Gibson DL, Gonzalez A, Gorlick K, Guo J, Hillmann B, Holmes S, Holste H, Huttenhower C, Huttley GA, Janssen S, Jarmusch AK, Jiang L, Kaehler BD, Kang KB, Keefe CR, Keim P, Kelley ST, Knights D, Koester I, Kosciolek T, Kreps J, Langille MGI, Lee J, Ley R, Liu YX, Loftfield E, Lozupone C, Maher M, Marotz C, Martin BD, McDonald D, Mclver LJ, Melnik AV, Metcalf JL, Morgan SC, Morton JT, Naimey AT, Navas-Molina JA, Nothias LF, Orchanian SB, Pearson T, Peoples SL, Petras D, Preuss ML, Pruesse E, Rasmussen LB, Rivers A, Robeson MS, Rosenthal P, Segata N, Shaffer M, Shiffer A, Sinha R, Song SJ, Spear JR, Swafford AD, Thompson LR, Torres PJ, Trinh P, Tripathi A, Turnbaugh PJ, Ul-Hasan S, van der Hooft JJJ, Vargas F, Vázquez-Baeza Y, Vogtmann E, von Hippel M, Walters W, Wan Y, Wang M, Warren J, Weber KC, Williamson CHD, Willis AD, Xu ZZ, Zaneveld JR, Zhang Y, Zhu Q, Knight R, and Caporaso JG. 2019.

Reproducible, interactive, scalable and extensible microbiome data science using QIIME 2. Nature Biotechnology 37: 852-857. https://doi.org/10.1038/s41587-019-0209-9

[29] Faith, Daniel P, 'Conservation Evaluation and Phylogenetic Diversity', Biological Conservation, 61 (1992), 1-10 <https://doi.org/10.1016/0006-3207(92)91201-3>

[30] Pielou, E. (1966). "The measurement of diversity in different types of biological collections". J. Theor. Biol. (13): 131144.

[31] Shannon, C.E. and Weaver, W. (1949). "The mathematical theory of communication". University of Illinois Press, Champaign, Illinois.

[32] Kruskal, William H, and W Allen Wallis, 'Use of Ranks in One-Criterion Variance Analysis', Journal of the American Statistical Association, 47 (1952), 583-621. 
[33] Weiss, Sophie, Zhenjiang Zech Xu, Shyamal Peddada, Amnon Amir, Kyle Bittinger, Antonio Gonzalez, and others, 'Normalization and Microbial Differential Abundance Strategies Depend upon Data Characteristics', Microbiome, 5 (2017), 27 <https://doi.org/10.1186/s40168-017-0237-y>

[34] Sorenson, T. (1948) "A method of establishing groups of equal amplitude in plant sociology based on similarity of species content." Kongelige Danske Videnskabernes Selskab 5.1-34: 4-7.

[35] Lozupone, C. and Knight, R. (2005). "UniFrac: a new phylogenetic method for comparing microbial communities." Applied and environmental microbiology 71 (12): 8228-8235.

[36] RStudio Team (2020). RStudio: Integrated Development for R. RStudio, PBC, Boston, MA URL http://www.rstudio.com/.

[37] Wickham et al., (2019). Welcome to the tidyverse. Journal of Open Source Software, 4(43), 1686, https://doi.org/10.21105/joss.01686

[38] Bisanz JE (2018). "qiime2R: Importing QIIME2 artifacts and associated data into R sessions." v0.99, <URL: https://github.com/jbisanz/qiime2R>.

\section{Figures}

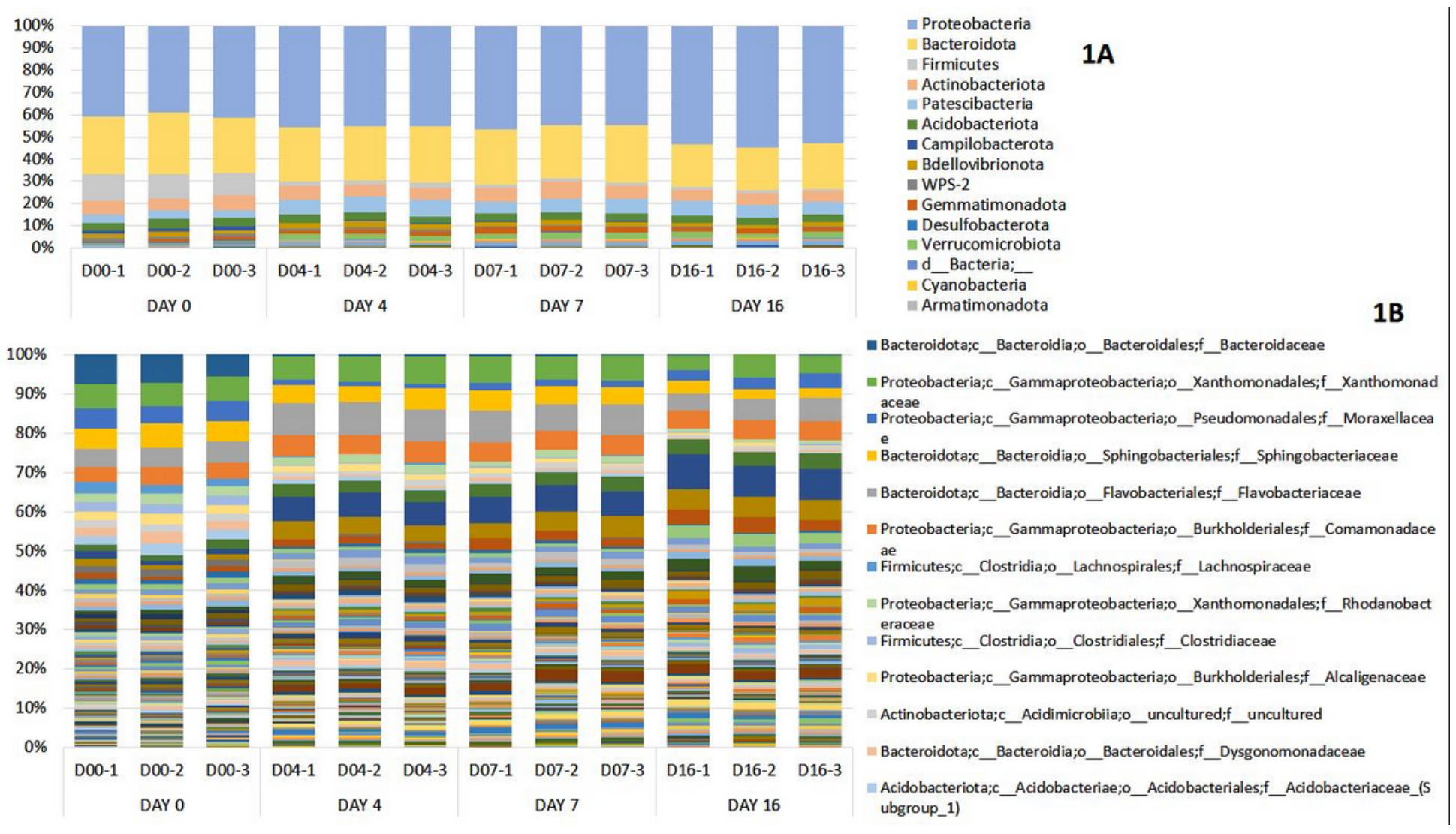

\section{Figure 1}

Barplot with the Bacterial 16S metataxonomical classification. Bacterial 16S metataxonomical classification by days at Phylum level (1A) and at Family level (1B). 


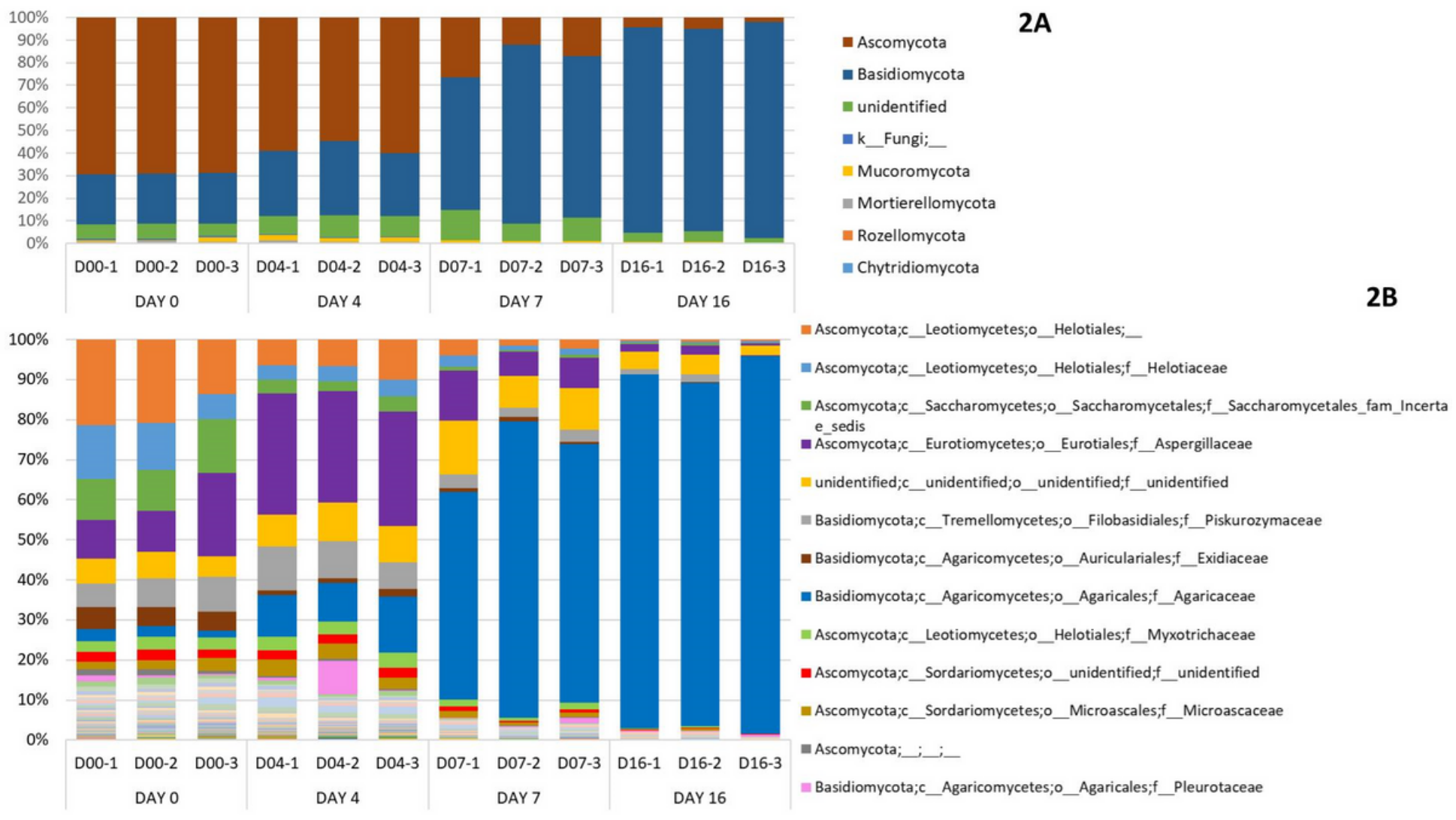

Figure 2

Barplot with the Fungal ITS2 metataxonomical classification. Fungal ITS2 metataxonomical classification by days at Phylum level (2A) and at Family level. (2B).

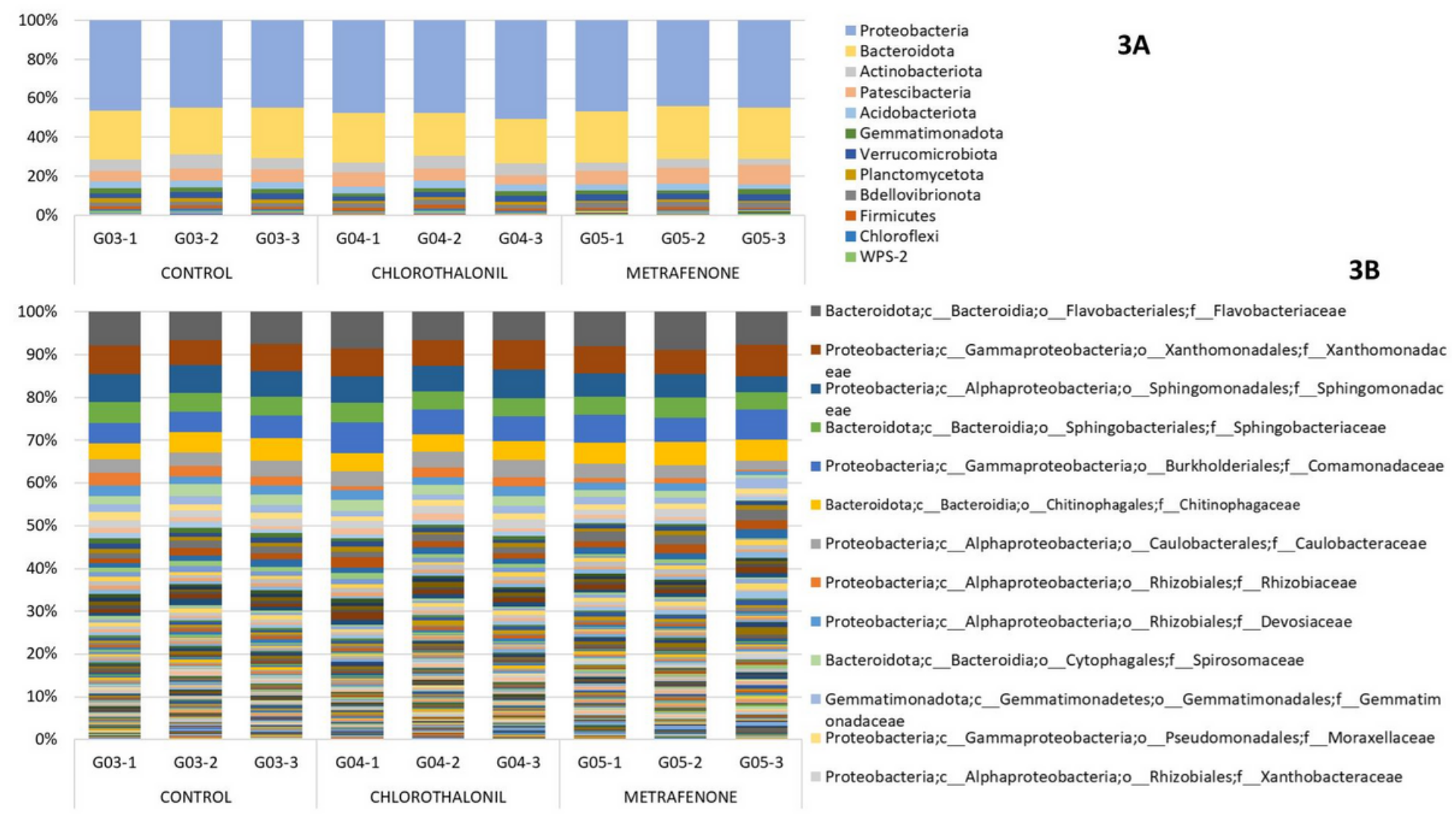




\section{Figure 3}

Barplot with the Bacterial 16S metataxonomical classification. Bacterial 16S metataxonomical classification by treatments at Day 7 at Phylum level (3A) and at Family level (3B).

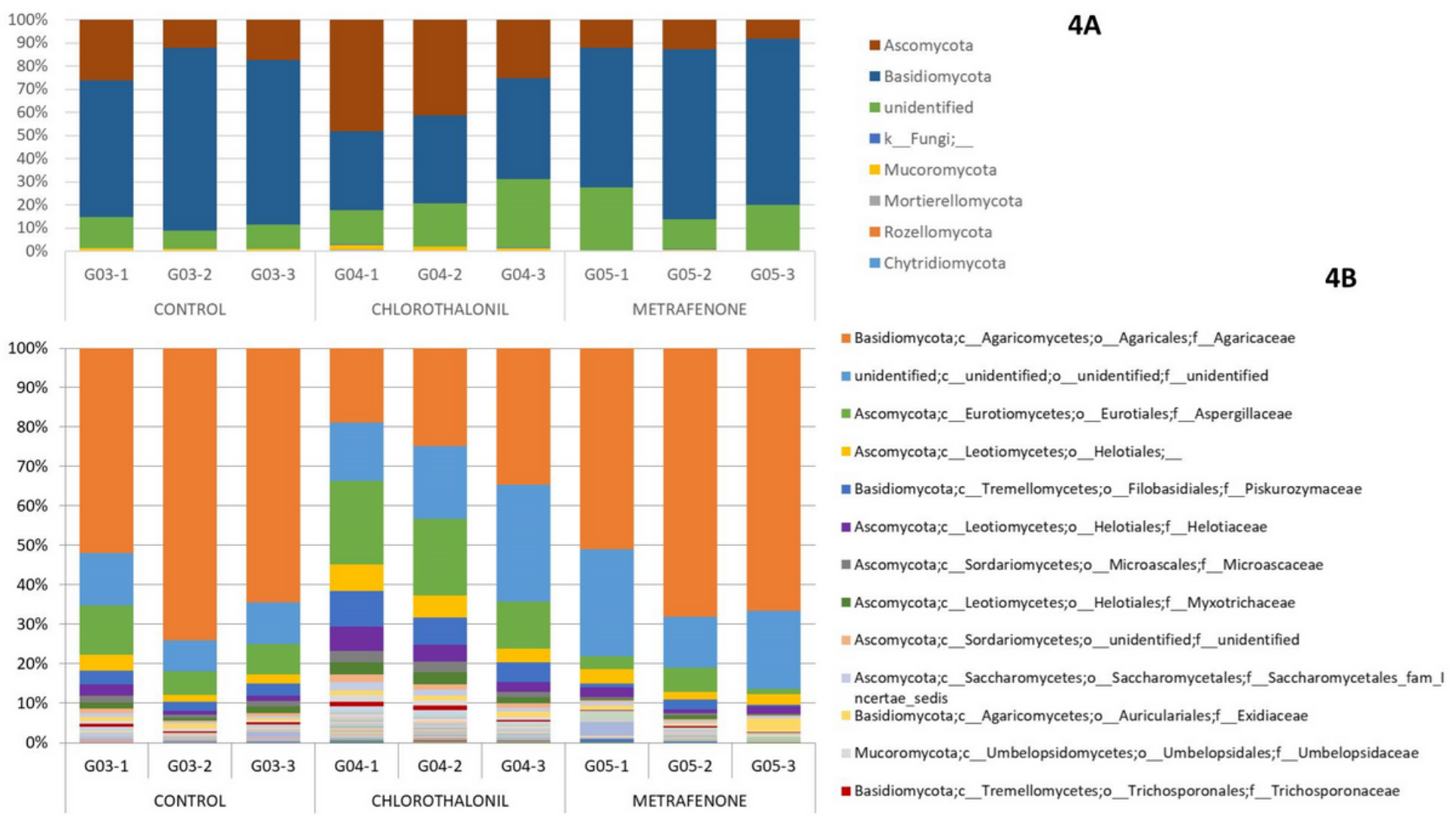

\section{Figure 4}

Barplot with the Fungal ITS2 metataxonomical classification. Fungal ITS2 metataxonomical classification by treatments at Day 7 at Phylum level (4A) and at Family level (4B). 


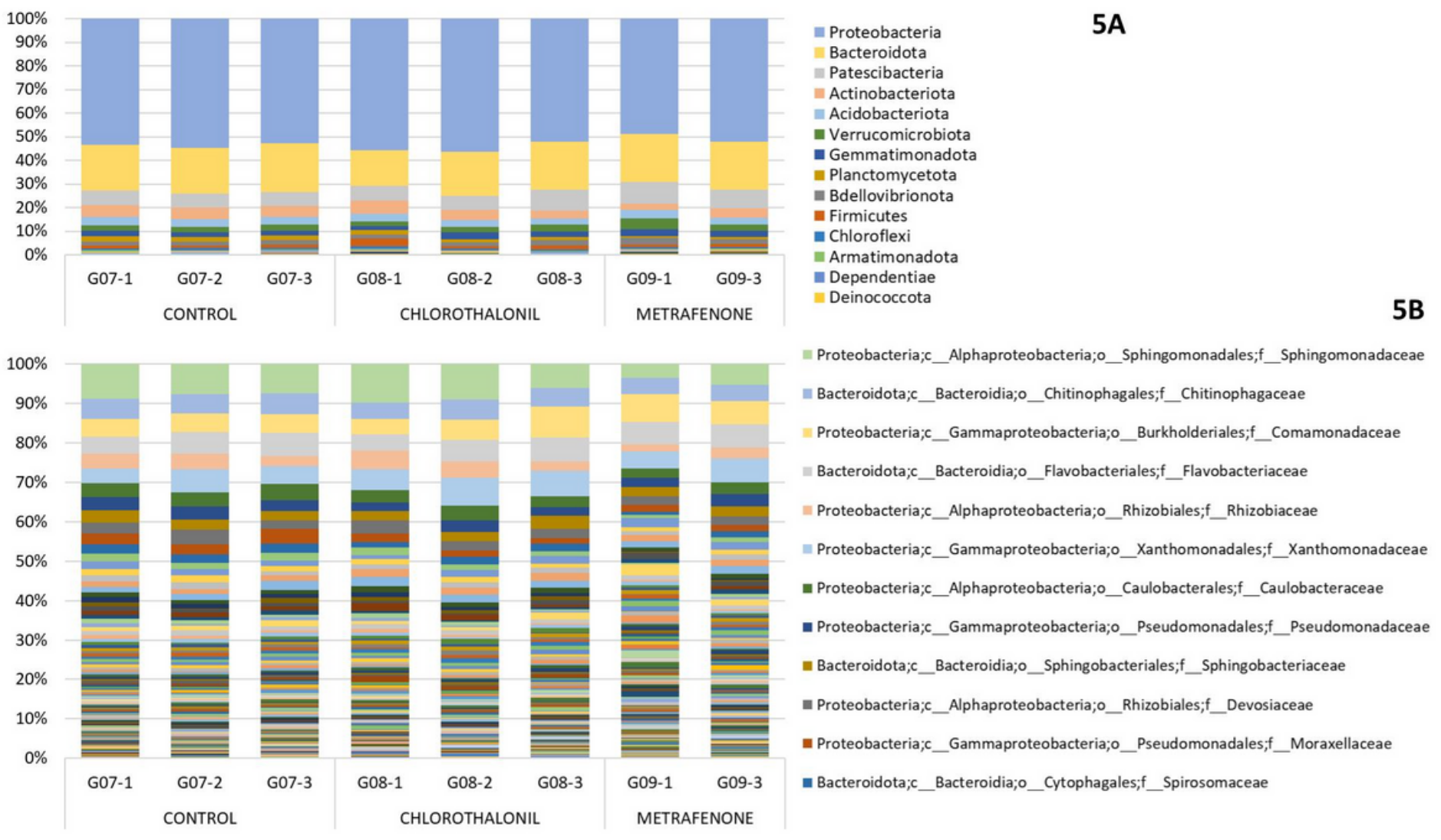

Figure 5

Barplot with the Bacterial 16S metataxonomical classification. Bacterial 16S metataxonomical classification by treatments at Day 16 at Phylum level (5A) and at Family level (5B).
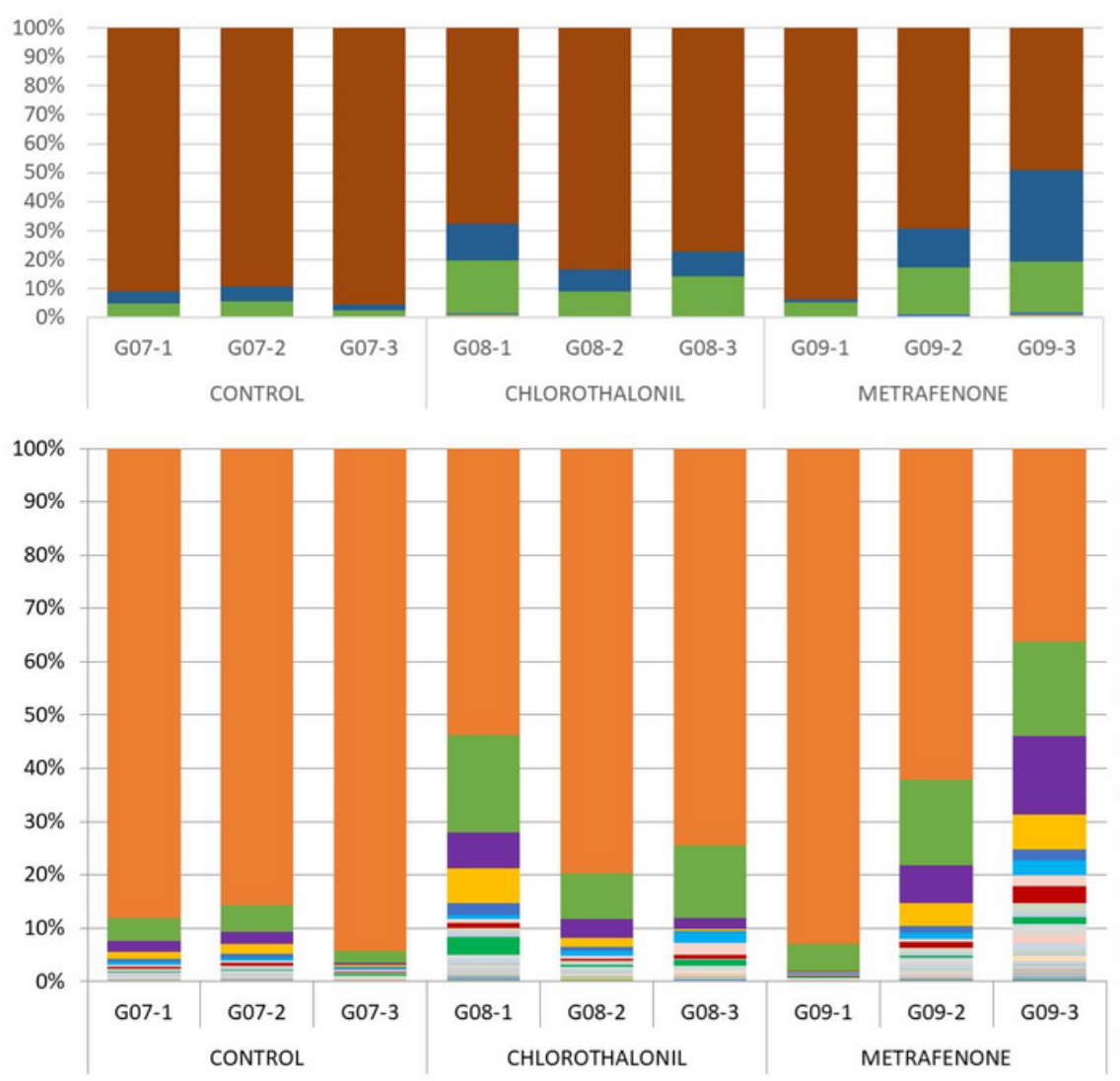

$6 A$

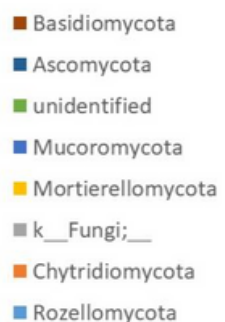

nozellomycota

6B

_Basidiomycota;c_Agaricomycetes;o_Agaricales;f_Agaricaceae

m unidentified;c_unidentified;o__unidentified;f__unidentified

- Ascomycota;c_Eurotiomycetes;o_Eurotiales,f__Aspergillaceae

= Basidiomycota;c_Tremellomycetes;o__Filobasidiales;f_Piskurozymaceae

Basidiomycota;c_Tremellomycetes;o_Trichosporonales;f_Trichosporonaceae

E Ascomycota;c_Leotiomycetes;o__Helotiales;_

Ascomycota;c_Leotiomycetes;o__Helotiales;f__Helotiaceae

- Ascomycota;c_Saccharomycetes;0_Saccharomycetales;f_Saccharomycetales_fa m_Incertae_sedis

Ascomycota;c_Sordariomycetes;o__Microascales;f_Microascaceae

Inucoromycota;c_Umbelopsidomycetes;o_Umbelopsidales;f_Umbelopsidaceae

= Basidiomycota;c_Agaricomycetes;o_Agaricales;f__Pleurotaceae

Ascomycota;c_Leotiomycetes; ;__Helotiales;f_Myxotrichaceae

Ascomycota;c_Sordariomycetes; 0 _Sordariales;f_Chaetomiaceae 


\section{Figure 6}

Barplot with the Fungal ITS2 metataxonomical classification. Fungal ITS2 metataxonomical classification by treatments at Day 16 at Phylum level (6A) and at Family level (6B).

\section{Supplementary Files}

This is a list of supplementary files associated with this preprint. Click to download.

- SupplementaryFigure1.jpg

- SupplementaryFigure2.jpg

- SupplementaryFigure3.jpg

- SupplementaryFigure4.jpg

- SupplementaryFigure5.jpg

- SupplementaryFigure6.jpg

- SupplementaryFigure7.jpg

- SupplementaryFigure8.jpg

- SupplementaryFigure9.jpg

- SupplementaryFigure10.jpg

- SupplementaryFigure11.jpg

- SupplementaryFigure12.jpg

- SupplementaryFigure13.jpg

- SupplementaryFigure14.jpg

- SupplementaryMaterialTitles.docx

- TableS1.xlsx

- Tables2.xlsx

- TableS3.xlsx 